\title{
Remote ischemic preconditioning impairs ventricular function and increases infarct size after prolonged ischemia in the isolated neonatal rabbit heart
}

\author{
Michael R. Schmidt, MD, PhD, ${ }^{a}$ Nicolaj B. Støttrup, MD, PhD, ${ }^{a}$ Marie M. Michelsen, MD, ${ }^{a}$ \\ Hussain Contractor, MD, PhD, ${ }^{b}$ Keld E. Sørensen, MD, ${ }^{a}$ Rajesh K. Kharbanda, MD, PhD, ${ }^{b}$ \\ Andrew N. Redington, MB, BS, MRCP(UK), MD, FRCP (UK), FRCP (C), ${ }^{\mathrm{c}}$ and Hans E. Bøtker, MD, PhD ${ }^{\mathrm{a}}$
}

\begin{abstract}
Objectives: Remote ischemic preconditioning (rIPC) reduces myocardial injury in adults and children undergoing cardiac surgery. We compared the effect of rIPC in adult and neonatal rabbits to investigate whether protection against ischemia-reperfusion injury can be achieved in the newborn heart by (1) in vivo rIPC and (2) dialysate from adult rabbits undergoing rIPC.
\end{abstract}

\begin{abstract}
Methods: Isolated hearts from newborn and adult rabbits were randomized into 3 subgroups (control, in vivo rIPC, and dialysate obtained from adult, remotely preconditioned rabbits). Remote preconditioning was induced by four 5-minute cycles of lower limb ischemia. Left ventricular (LV) function was assessed using a balloontipped catheter, glycolytic flux by tracer kinetics, and infarct size by tetrazolium staining. Isolated hearts underwent stabilization while perfused with standard Krebs-Henseleit buffer (control and in vivo rIPC) or Krebs-Henseleit buffer with added dialysate, followed by global no-flow ischemia and reperfusion.
\end{abstract}

Results: Within the age groups, the baseline LV function was similar in all subgroups. In the adult rabbit hearts, rIPC and rIPC dialysate attenuated glycolytic flux and protected against ischemia-reperfusion injury, with better-preserved LV function compared with that of the controls. In contrast, in the neonatal hearts, the glycolytic flux was lower and LV function was better preserved in the controls than in the rIPC and dialysate groups. In the adult hearts, the infarct size was reduced in the rIPC and dialysate groups compared with that in the controls. In the neonatal hearts, the infarct size was smaller in the controls than in the rIPC and dialysate groups.

Conclusions: Remote ischemic preconditioning does not protect against ischemia-reperfusion injury in isolated newborn rabbit hearts and might even cause deleterious effects. Similar adverse effects were induced by dialysate from remotely preconditioned adult rabbits. (J Thorac Cardiovasc Surg 2014;147:1049-55)

Impaired ventricular systolic and diastolic function is an important contributor to the mortality and morbidity associated with pediatric cardiac surgery, ${ }^{1,2}$ particularly after complex surgery performed in the neonate and young infant. The immature heart is structurally, functionally, and metabolically different from the adult heart and undergoes fundamental changes around the time of birth; however, our understanding of the detailed ontogeny of these changes remains incomplete.

\footnotetext{
From the Department of Cardiology, ${ }^{\mathrm{a}}$ Aarhus University Hospital, Skejby, Brendstrupgaardsvej, Aarhus N, Aarhus, Denmark; Department of Cardiology, ${ }^{\text {b John }}$ Radcliffe Hospital, Headington, Oxford, United Kingdom; and Division of Cardiology, ${ }^{\mathrm{c}}$ Hospital for Sick Children, Toronto, Ontario, Canada.

The project was funded by unrestricted grants from Fondation Leducq and the Danish Heart Foundation.

Disclosures: Michael R. Schmidt, Rajesh K. Kharbanda, Andrew N. Redington, and Hans Erik Bøtker are shareholders in CellAegis Devices. All other authors have nothing to disclose with regard to commercial support.

Received for publication April 7, 2013; revisions received May 10, 2013; accepted for publication May 24, 2013; available ahead of print July 22, 2013

Address for reprints: Michael R. Schmidt, MD, PhD, Department of Cardiology, Aarhus University Hospital, Skejby, Brendstrupgaardvej, Rosenvangs Alle 218-234, Aarhus N, Aarhus 8200 Denmark (E-mail: Michael.rahbek@ki.au.dk). $0022-5223 / \$ 36.00$

Copyright (c) 2014 by The American Association for Thoracic Surgery http://dx.doi.org/10.1016/j.jtcvs.2013.05.022
}

Identification of the physiologic differences in the developing myocardium is a prerequisite to understanding the underlying mechanisms of myocardial protection and devising new strategies for protecting the immature heart during cardiac surgery.

Remote ischemic preconditioning (rIPC), induced by cycles of ischemia and reperfusion of a limb before surgery, has recently emerged as a potential method of averting myocardial injury during cardiopulmonary bypass in older childre $^{3}$ and adults ${ }^{4}$ undergoing cardiac surgery; however, no studies are available of its effectiveness in the immature heart. This is important, because evidence from experimental studies of local ischemic preconditioning, in which the cycles of ischemia and reperfusion were applied directly to the tissue subjected to prolonged ischemia, has shown that the responses might display distinct maturational dependence. For example, some studies have shown that the ability to achieve cardioprotection from local ischemic preconditioning is not present in fetal life and only becomes apparent after 1 to 4 weeks (depending on the species) after birth. $^{5-7}$ However, others have shown effective pharmacologic and ischemic preconditioning in immature rabbit hearts ${ }^{8}$ and isolated neonatal cardiomyocytes. ${ }^{9,10}$ 


$$
\begin{aligned}
& \text { Abbreviations and Acronyms } \\
& \begin{aligned}
\mathrm{IR} & =\text { ischemia-reperfusion } \\
\mathrm{dP} / \mathrm{dt}_{\max } & =\text { maximal } \mathrm{dP} / \mathrm{dt} \\
\mathrm{dP} / \mathrm{dt}_{\min } & =\text { minimal } \mathrm{dP} / \mathrm{dt} \\
\mathrm{KH} & =\text { Krebs-Henseleit } \\
\mathrm{LV} & =\text { left ventricular } \\
\mathrm{LVDP} & =\mathrm{LV} \text { developed pressure } \\
\mathrm{rIPC} & =\text { remote ischemic preconditioning }
\end{aligned}
\end{aligned}
$$

Given the contrasting data, it is unsurprising that the mechanisms underlying "preconditioning resistance" remain unknown, although the immature myocardium consistently appears intrinsically more resistant to hypoxia and possibly ischemia, ${ }^{6,11}$ raising the possibility that it is already "preconditioned" in some way.

Moreover, the transition from immature, predominantly carbohydrate-dependent myocardial metabolism to the mature "omnivore" myocardium occurs-with some species variation-within the first weeks of life. ${ }^{12}$ Also, although local ischemic preconditioning attenuates glycolytic flux in the mature myocardium, ${ }^{13}$ it is unknown whether this effect is present and beneficial in the carbohydrate-dependent immature myocardium.

Nonetheless, the relevance of these studies to rIPC is questionable, because rIPC does not involve ischemic bursts of the myocardium itself to induce the preconditioned state. However, in an excellent recent study by Jones et al, ${ }^{14}$ no detectable cardioprotection was achieved by rIPC in hypoxic neonates undergoing cardiopulmonary bypass surgery.

We have recently shown that rIPC liberates a small $(<15$ $\mathrm{kDa}$ ), hydrophobic cardioprotective factor into the bloodstream, which is dialyzable from plasma and transferable from rabbit to rabbit to protect against ischemiareperfusion (IR) injury in Langendorff preparations. ${ }^{15}$

The purpose of the present study was to use the unique properties of rIPC to study the following questions:

1. Does rIPC protect the isolated newborn rabbit heart from IR injury?

2. Does dialysate from adult rabbits undergoing rIPC protect the isolated newborn rabbit heart from IR injury?

\section{METHODS \\ Design}

IR injury was studied in isolated hearts from 24 newborn (1-2 days old, randomized to 3 groups of 8 animals) and 24 adult rabbits ( 3 months old, randomized to 3 groups of 8 animals). An additional 16 adult rabbits were used as donors of blood after undergoing rIPC. Blood from 1 adult rabbit provided enough dialysate for 1 isolated newborn or adult. Consequently, 6 groups were studied:

1. Adult hearts subjected to IR injury alone (see below for details)

2. Adult hearts subjected to IR injury after in vivo rIPC
3. Adult hearts subjected to IR injury after perfusion with dialysate from a donor rabbit undergoing rIPC

4. Newborn hearts subjected to IR injury alone (see below for details)

5. Newborn hearts subjected to IR injury after in vivo rIPC

6. Newborn hearts subjected to IR injury after perfusion with dialysate from an adult donor rabbit undergoing rIPC

\section{Rabbits}

The rabbits were handled according to the guidelines appointed by the Danish Committee for Animal Research (Dyreforsøgstilsynet, Copenhagen, Denmark). Adult (3-kg), male, white, New Zealand rabbits were housed in single cages and provided with access to food and water ad libitum. Newborn (aged 2-4 days; weight, 150-200 g), white New Zealand rabbits were kept with their mother until the time of study.

\section{Anesthesia and Ventilation}

In brief, all the rabbits (neonatal and adult) were anesthetized with a subcutaneous injection of a mixture of midazolam (Dormicum) and etomidate (Hypnorm). When an adequate depth of anesthesia had been confirmed, a tracheotomy was performed, and the rabbit was mechanically ventilated (Ugo Basile, Comerio, Italy).

\section{rIPC Protocol}

The rabbits used as blood donors and the rabbits in the in vivo rIPC groups underwent the same preconditioning protocol. After sedation and intubation, rIPC was performed using tourniquet occlusion of the lower left limb for 4 cycles of 5 minutes of ischemia followed by 5 minutes of reperfusion (40 minutes total). The rabbit remained sedated and ventilated for an additional 20 minutes before the heart was explanted or blood was taken for dialysis.

\section{Blood Sampling and Dialysis Preparation}

The rabbits used as blood donors had a 5F sheath inserted into the right common carotid artery. Blood was rapidly withdrawn through the sheath for a period of 2 minutes to avoid hemodynamic disturbance. The adult rabbits yielded approximately $150 \mathrm{~mL}$ of whole blood that was collected in heparinized tubes and spun at $3000 \mathrm{rpm}$ for 20 minutes at $4^{\circ} \mathrm{C}$. The plasma was separated and dialyzed against sterile water through a $15-\mathrm{kDa}$ Spectrapor dialysis membrane (Spectrum Laboratories, Rancho Dominguez, Calif) for 12 hours at $4^{\circ} \mathrm{C}$. The dialysate was subsequently diluted to provide a $10 \%$ concentration in a standard KrebsHenseleit (KH) buffer (see below). The dialysate was divided into equal portions, supplying the substrate needed for 1 neonatal and 1 adult isolated heart.

\section{Nonworking Langendorff IR Injury Protocol}

A laparotomy and thoracotomy were performed, and the heart was dissected free from the surrounding structures. A bolus of $1000 \mathrm{IU} / \mathrm{kg}$ heparin (Leo Pharma, Copenhagen, Denmark) was given through the femoral vein. The heart was cannulated in situ, mounted on the Langendorff apparatus, and perfused retrogradely with $\mathrm{KH}$ buffer $(\mathrm{NaCl} 118.5 \mathrm{mmol} / \mathrm{L}$, $\mathrm{KCl} 4.7 \mathrm{mmol} / \mathrm{L}, \mathrm{NaHCO}_{3} 25.0 \mathrm{mmol} / \mathrm{L}$, glucose monohydrate 11.1 $\mathrm{mmol} / \mathrm{L}, \mathrm{MgSO}_{4}, 7 \mathrm{H} 2 \mathrm{O} 1.2 \mathrm{mmol} / \mathrm{L}, \mathrm{CaCl}_{2} 2.4 \mathrm{mmol} / \mathrm{L}$, and $\mathrm{KH}_{2} \mathrm{PO}_{4}$ $1.2 \mathrm{mmol} / \mathrm{L}$; Sigma Aldrich, St Louis, Mo) at a pressure of $70 \mathrm{~mm} \mathrm{Hg}$. The perfusion solution was fully oxygenated with $95 \%$ oxygen and $5 \%$ carbon dioxide and maintained at $37^{\circ} \mathrm{C}$. The hearts were allowed to stabilize for 20 minutes. For the next 35 minutes, the hearts in groups 3 and 6 were switched to $\mathrm{KH}$ buffer with $10 \%$ adult dialysate, and the hearts in the other groups were perfused only with standard $\mathrm{KH}$ buffer. All hearts were then subjected to 40 minutes of normothermic global no-flow ischemia and subsequently 120 minutes of reperfusion with standard $\mathrm{KH}$ solution. 


\section{Left Ventricular Function}

Left ventricular (LV) function was measured with a balloon catheter placed in the left ventricle through the mitral valve and connected to a pressure transducer. The data were sampled using a high-fidelity analog to digital hardware to dedicated data acquisition software (Notocord, Croissy Sur Seine, France). The derived indexes of systolic (maximal $\mathrm{dP} / \mathrm{dt}\left[\mathrm{dP} / \mathrm{dt}_{\max }\right]$ ) and diastolic (minimal $\mathrm{dP} / \mathrm{dt}\left[\mathrm{dP} / \mathrm{dt}_{\min }\right]$ ) function were calculated automatically by the software program.

\section{Assessment of Myocardial Infarction}

At the end of the experiment, the hearts were frozen at $-80^{\circ} \mathrm{C}$ for 15 minutes, subsequently cut into $1.5-\mathrm{mm}$ slices, and immersed in $1 \%$ 2,3,5-triphenyltetrazolium chloride (Sigma Aldrich) at $37^{\circ} \mathrm{C}$ for 3 minutes at $\mathrm{pH}$ 7.4. The hearts were then stored overnight in $2 \mathrm{~mL}$ of Lillie's solution ( $4 \%$ formaldehyde buffer; VWR International, Herlev, Denmark) to enhance the color contrast. The next day, each heart was weighed and scanned with a flatbed scanner (HP ScanJet 4300C; Hewlett Packard, Palo Alto, Calif). The area of the left ventricle and the area of infarction were assessed semiautomatically by computer planimetry (UTHSCSA Image Tool for Windows, version 3.0, San Antonio, Tex). The infarct size was calculated by summing the percentage of infarction from each slice, expressed as a function of slice weight. All analyses were performed by a blinded observer.

\section{Tracer Kinetics}

Glycolytic flux from exogenous glucose was measured using statically labeled tritiated D-[5- $\left.{ }^{3} \mathrm{H}\right]$ glucose (PerkinElmer, Waltham, Mass) added to $\mathrm{KH}$ buffer at a mixture of $10 \mu \mathrm{Ci} / 200 \mathrm{~mL}$ perfusate. The specific activity of glucose $(-5 \mu \mathrm{Ci} / \mathrm{mmol})$ was determined by counting the emission from an aliquot of perfusate collected at the beginning of each experiment. The flux of exogenous glucose to pyruvate was assessed by the measurement of tritiated ${ }^{3} \mathrm{H}_{2} \mathrm{O}$ production at the site of enolase. ${ }^{16}$

Aliquots of coronary effluent were sampled every 5 minutes, and the metabolic rate calculations were corrected for "arterial" aliquots sampled from the bottom of the oxygenator. The separation of tritiated glucose from ${ }^{3} \mathrm{H}_{2} \mathrm{O}$ was performed using anion exchange chromatography on an $\mathrm{AG}$

TABLE 1. Hemodynamic parameters

\begin{tabular}{|c|c|c|c|c|c|c|c|}
\hline \multirow[b]{2}{*}{ Variable } & \multicolumn{3}{|c|}{ Before ischemia } & \multicolumn{4}{|c|}{ Reperfusion } \\
\hline & Baseline & $40 \mathrm{~min}$ & $\begin{array}{c}P \text { value } \\
\text { (vs control) }\end{array}$ & $10 \mathrm{~min}$ & $30 \mathrm{~min}$ & $120 \mathrm{~min}$ & $\begin{array}{c}P \text { value } \\
\text { (vs control) }\end{array}$ \\
\hline \multicolumn{8}{|l|}{ LVDP (mm Hg) } \\
\hline Neonatal control & $48.8 \pm 3.2$ & $41.2 \pm 3.6$ & & $21.1 \pm 2.5$ & $31.0 \pm 2.3$ & $24.0 \pm 2.2$ & \\
\hline Neonatal rIPC & $37.5 \pm 8.5$ & $36.4 \pm 8.0$ & $<.05$ & $11.3 \pm 2.3$ & $14.9 \pm 2.5$ & $11.0 \pm 2.0$ & $<.01$ \\
\hline Neonatal rIPC dialysate & $43.9 \pm 3.4$ & $31.5 \pm 3.6$ & $<.01$ & $9.6 \pm 2.5$ & $13.3 \pm 2.3$ & $8.0 \pm 2.2$ & $<.01$ \\
\hline Adult control & $115.5 \pm 7.8$ & $113.6 \pm 6.8$ & & $4.9 \pm 1.4$ & $9.1 \pm 2.2$ & $8.8 \pm 2.3$ & \\
\hline Adult rIPC & $109.7 \pm 6.0$ & $110.1 \pm 6.5$ & NS & $17.2 \pm 6.4$ & $21.1 \pm 3.5$ & $25.4 \pm 4.6$ & $<.01$ \\
\hline Adult rIPC dialysate & $115.3 \pm 18.1$ & $116.1 \pm 11.0$ & NS & $20.6 \pm 5.7$ & $23.3 \pm 4.7$ & $30.8 \pm 5.6$ & $<.01$ \\
\hline \multicolumn{8}{|l|}{ HR (beats/min) } \\
\hline Neonatal control & $195.5 \pm 11.3$ & $218.1 \pm 13.7$ & & $195.5 \pm 11.3$ & $228.1 \pm 11.5$ & $200.2 \pm 14.3$ & \\
\hline Neonatal rIPC & $228.1 \pm 11.5$ & $228.1 \pm 23.1$ & NS & $205.1 \pm 20.8$ & $225.4 \pm 19.5$ & $223.4 \pm 19.0$ & NS \\
\hline Neonatal rIPC dialysate & $214.1 \pm 20.2$ & $212.8 \pm 21.8$ & NS & $212.8 \pm 5.0$ & $219.9 \pm 21.3$ & $203.8 \pm 19.4$ & NS \\
\hline Adult control & $187.3 \pm 8.0$ & $172.6 \pm 7.9$ & & $196.1 \pm 30.7$ & $212.5 \pm 35.2$ & $181.5 \pm 20.4$ & \\
\hline Adult rIPC & $174.2 \pm 5.8$ & $157.9 \pm 9.6$ & $<.05$ & $180.2 \pm 31.6$ & $151.6 \pm 11.2$ & $147.4 \pm 6.3$ & $<.05$ \\
\hline Adult rIPC dialysate & $214.0 \pm 15.4$ & $186.1 \pm 18.6$ & $<.05$ & $174.1 \pm 11.9$ & $157.3 \pm 27.0$ & $185.6 \pm 5.2$ & NS \\
\hline \multicolumn{8}{|l|}{$\mathrm{dP} / \mathrm{dt}_{\min }(\mathrm{mm} \mathrm{Hg} / \mathrm{s})$} \\
\hline Neonatal control & $-1310.3 \pm 241.9$ & $-1044.3 \pm 153.4$ & & $-507.1 \pm 167.5$ & $-683.9 \pm 109.2$ & $-567.3 \pm 127.8$ & \\
\hline Neonatal rIPC & $-775.8 \pm 185.3$ & $-812.8 \pm 166.7$ & $<.01$ & $-261.4 \pm 30.0$ & $-329.4 \pm 34.6$ & $-288.8 \pm 36.1$ & $<.01$ \\
\hline Neonatal rIPC dialysate & $-855.5 \pm 127.9$ & $-634.7 \pm 175.3$ & $<.001$ & $-202.6 \pm 23.6$ & $-270.3 \pm 66.0$ & $-205.4 \pm 70.0$ & $<.01$ \\
\hline Adult control & $-1576.0 \pm 105.8$ & $-1580.2 \pm 105.2$ & & $-266.9 \pm 53.6$ & $-368.3 \pm 81.6$ & $-398.9 \pm 70.5$ & \\
\hline Adult rIPC & $-1464.0 \pm 69.8$ & $-1537.1 \pm 149.3$ & NS & $-610.1 \pm 171.9$ & $-518.8 \pm 94.1$ & $-616.0 \pm 124.3$ & $<.01$ \\
\hline Adult rIPC dialysate & $-1645.4 \pm 176.4$ & $-1758.5 \pm 158.7$ & NS & $-662.7 \pm 126.8$ & $-368.3 \pm 81.6$ & $-398.9 \pm 70.5$ & $<.01$ \\
\hline \multicolumn{8}{|l|}{$\mathrm{dP} / \mathrm{dt}_{\max }(\mathrm{mm} \mathrm{Hg} / \mathrm{s})$} \\
\hline Neonatal control & $930.3 \pm 193.1$ & $884.9 \pm 234.0$ & & $420.7 \pm 193.2$ & $567.8 \pm 267.5$ & $475.8 \pm 251.9$ & \\
\hline Neonatal rIPC & $914.5 \pm 261.7$ & $744.3 \pm 195.7$ & NS & $311.7 \pm 50.4$ & $324.7 \pm 34.6$ & $257.5 \pm 28.8$ & $<.05$ \\
\hline Neonatal rIPC dialysate & $774.0 \pm 397.9$ & $533.7 \pm 290.6$ & NS & $252.3 \pm 107.5$ & $312.9 \pm 102.6$ & $230.4 \pm 145.5$ & $<.05$ \\
\hline Adult control & $1994.4 \pm 282.0$ & $2128.3 \pm 185.5$ & & $261.5 \pm 51.3$ & $369.5 \pm 89.6$ & $408.1 \pm 77.2$ & \\
\hline Adult rIPC & $1748.9 \pm 162.1$ & $1825.6 \pm 192.0$ & NS & $567.1 \pm 127.8$ & $541.1 \pm 94.3$ & $617.2 \pm 99.1$ & $<.01$ \\
\hline Adult rIPC dialysate & $2186.6 \pm 288.4$ & $2139.5 \pm 318.8$ & NS & $640.4 \pm 115.3$ & $647.7 \pm 141.0$ & $775.2 \pm 142.8$ & $<.01$ \\
\hline \multicolumn{8}{|l|}{ Coronary flow (mL/min) } \\
\hline Neonatal control & $11.7 \pm 3.2$ & $9.6 \pm 1.6$ & & $12.8 \pm 2.0$ & $12.8 \pm 1.5$ & $9.6 \pm 1.3$ & \\
\hline Neonatal rIPC & $11.1 \pm 0.6$ & $11.7 \pm 0.9$ & NS & $13.7 \pm 1.0$ & $13.0 \pm 0.9$ & $12.4 \pm 1.9$ & NS \\
\hline Neonatal rIPC dialysate & $8.7 \pm 1.5$ & $8.2 \pm 1.7$ & NS & $11.3 \pm 2.9$ & $10.3 \pm 1.3$ & $14.1 \pm 1.2$ & NS \\
\hline Adult control & $56.0 \pm 3.8$ & $53.0 \pm 4.3$ & & $35.1 \pm 6.0$ & $42.2 \pm 5.8$ & $33.5 \pm 5.7$ & \\
\hline Adult rIPC & $57.7 \pm 6.1$ & $54.9 \pm 6.0$ & NS & $45.6 \pm 6.9$ & $41.5 \pm 5.3$ & $31.5 \pm 6.6$ & $<.07$ \\
\hline Adult rIPC dialysate & $70.6 \pm 7.6$ & $61.7 \pm 3.8$ & NS & $50.5 \pm 5.3$ & $46.2 \pm 5.5$ & $40.6 \pm 8.4$ & $<.05$ \\
\hline
\end{tabular}

$L V D P$, Left ventricular developed pressure; $r I P C$, remote ischemic preconditioning; $N S$, not significant; $H R$, heart rate; $d P / d t_{\max }$, maximal $\mathrm{dP} / \mathrm{dt} ; d P / d t_{\min }$, minimal $\mathrm{dP} / \mathrm{dt}$. 

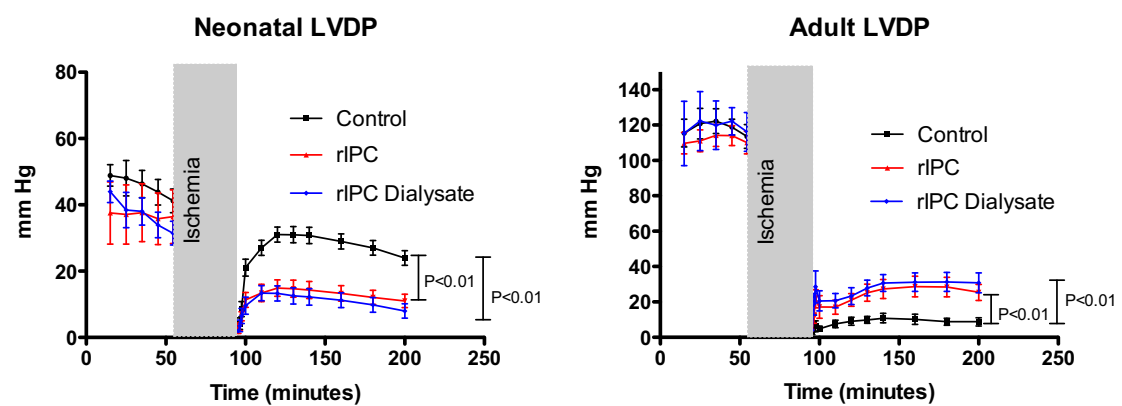

FIGURE 1. Left ventricular systolic function in neonatal and adult rabbit hearts. $L V D P$, Left ventricular developed pressure; $r I P C$, remote ischemic preconditioning.

1-X8 resin column (Bio-Rad Laboratories, Hercules, Calif). The ${ }^{3} \mathrm{H}_{2} \mathrm{O}$ was dissolved in $10 \mathrm{~mL}$ OptiPhase scintillation solution (Perkin-Elmer, Waltham, Mass), and the amount was quantified by $\beta$-scintillation counting on a TriCarb 2900TR liquid scintillation analyzer (Perkin-Elmer). The glycolytic flux during both stabilization and reperfusion was calculated by dividing the counts per minute by the specific activity of the glucose in the perfusate. The results were plotted against time, and the glycolytic flux rate was calculated.

\section{Statistical Analysis}

All values are presented as the mean \pm standard error of the mean. The LV developed pressure (LVDP) was calculated as the difference between the LV systolic pressure and LV diastolic pressure. Hemodynamic data were compared using 2-way analysis of variance, with repeated measures. One-way analysis of variance was used to compare the infarct size and hemodynamic parameters at dedicated measurement points (Table 1). The glycolytic flux data were assessed using 1-way analysis of variance of the area under the curve. $P=.05$ was considered statistically significant. Statistical analyses were performed using GraphPad Prism, version 5.01 (GraphPad Software, San Diego Calif).

\section{RESULTS}

A total of 39 newborn and 45 adult rabbits were used in this project, because 15 neonatal and 5 adult rabbits died of technical or surgical problems before inclusion in the study.

\section{Baseline Characteristics}

The hearts from the newborn rabbits did not differ among the groups at baseline with regard to the LVDP, heart rate, rate pressure-product, $\mathrm{dP} / \mathrm{dt}_{\min }, \mathrm{dP} / \mathrm{dt}_{\max }$, and $\mathrm{LV}$ end- diastolic pressure. Similarly, the , heart rate, $\mathrm{dP} / \mathrm{dt}_{\min }, \mathrm{dP} /$ $\mathrm{dt}_{\max }$, and LV end-diastolic pressure were equal at baseline in the hearts from the adult rabbits (Table 1). No differences in coronary perfusion between both neonatal and adult groups were observed throughout the study (Table 1).

\section{Function After rIPC}

In neonatal hearts, the LVDP (Figure 1), rate pressureproduct, and $\mathrm{dP} / \mathrm{dt}_{\max }$ were significantly higher during reperfusion in the controls than in the neonatal hearts treated with either in vivo rIPC or dialysate from remotely preconditioned adult rabbits, suggesting that rIPC impairs post-IR functional recovery (Table 1).

In the hearts from adult rabbits, the systolic and diastolic performance was similar before global ischemia; however, the hearts treated with either in vivo rIPC or dialysate from remotely preconditioned adult rabbits had significantly greater LVDPs, rate pressure-products, and $\mathrm{dP} / \mathrm{dt}_{\max }$ and lower $\mathrm{dP} / \mathrm{dt}_{\min }$, thus, showing the expected protection against systolic and diastolic dysfunction during reperfusion (Table 1 and Figure 1).

Neonatal hearts exposed to either in vivo rIPC or rIPC dialysate had a significantly lower $\operatorname{LVDP}(P<.05$ and $P<.01)$ than did the controls even before ischemia. Similarly, the $\mathrm{dP} / \mathrm{dt}_{\text {min }}$ was significantly higher in the treated neonatal groups than in the controls. In contrast, no differences were found in the LVDP, $\mathrm{dP} / \mathrm{dt}_{\max }$, and $\mathrm{dP} / \mathrm{dt}_{\min }$ among the adult groups before ischemia.

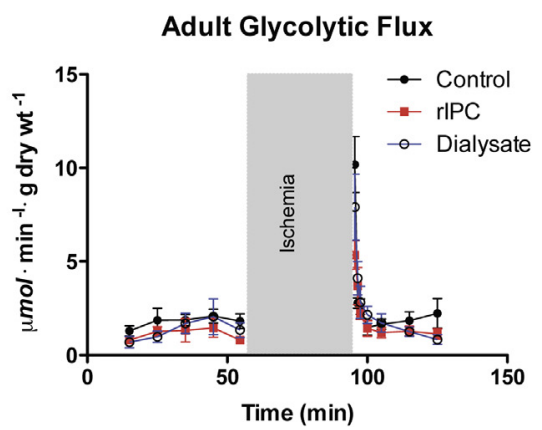

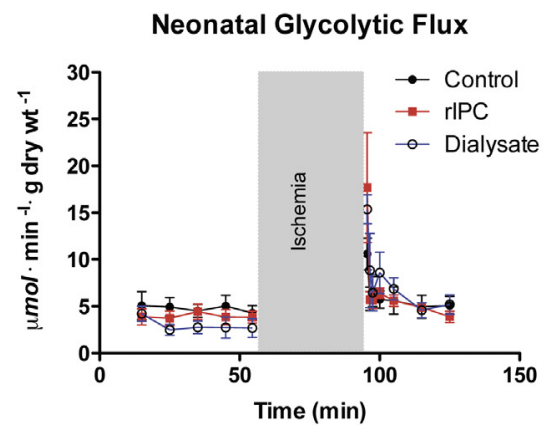

FIGURE 2. Glycolytic flux. $r I P C$, Remote ischemic preconditioning. 


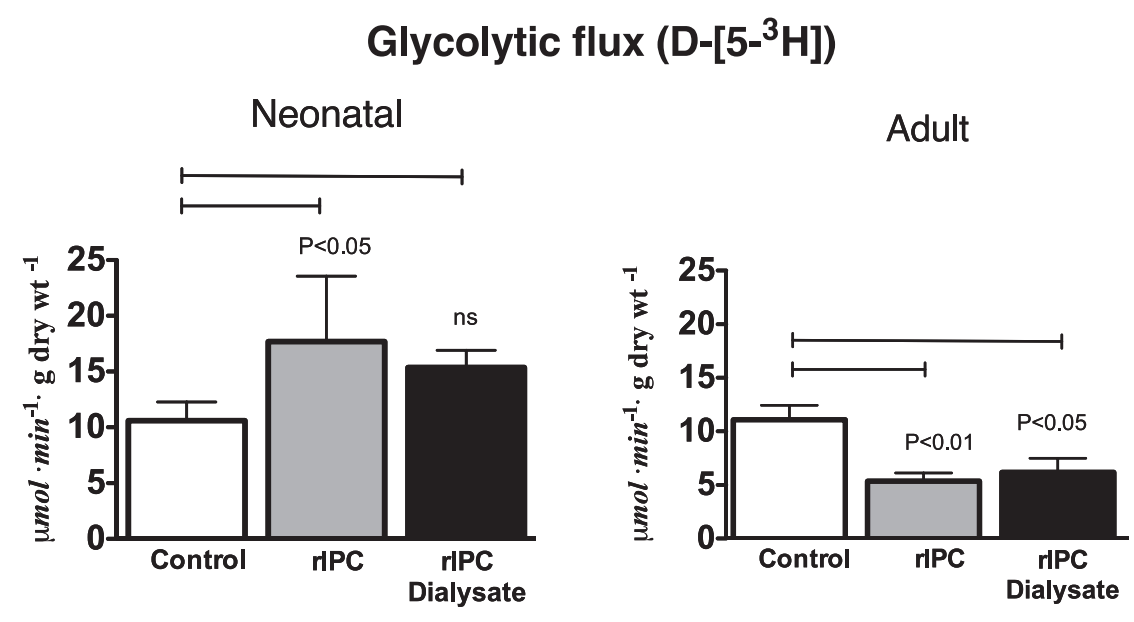

FIGURE 3. Glycolytic flux in early reperfusion. $r I P C$, Remote ischemic preconditioning.

\section{Metabolism}

Glycolytic flux was significantly greater in all neonatal groups than in the adult groups throughout the protocol (Figure 2). In neonatal hearts, no difference was found between groups before ischemia. However, in early reperfusion (the first 5 minutes), the neonatal hearts treated with in vivo rIPC had significantly higher glycolytic flux $(P<.01)$, with a trend toward higher flux in the hearts treated with adult rIPC dialysate $(P<.07$; Figure 3$)$.

In contrast, adult hearts treated with either in vivo rIPC or dialysate from remotely preconditioned adult rabbits showed significantly lower glycolytic flux during early reperfusion $(P<.01$ and $P<.05$, respectively; Figure 3$)$.

\section{Infarct Size}

The infarct size tended to be larger in the neonatal hearts treated with either in vivo $\operatorname{rIPC}(P=.06)$ and was significantly larger in the neonatal hearts treated with dialysate from remotely preconditioned adult rabbits $(P<.01)$ compared with the neonatal control hearts. In contrast, adult hearts treated with either in vivo rIPC or dialysate from remotely preconditioned adult rabbits had a significantly smaller infarct size than the controls (both $P<.01$; Figure 4).

The neonatal control hearts recovered significantly better after prolonged ischemia, with the LVDP after 2 hours of reperfusion reaching $49.9 \% \pm 22 \%$ of baseline compared with adult control hearts, which regained only $8.1 \% \pm$ $6.7 \%$ of baseline LVDP after 2 hours of reperfusion $(P<.01)$. The neonatal control hearts had a significantly smaller infarct size, with an infarct size/area at risk of $60.6 \% \pm 10.5 \%$, compared with an infarct size/area at risk of $81.8 \% \pm 7.8 \%$ in the adult control hearts $(P<.01)$.

\section{DISCUSSION}

The results of the present study have confirmed the lack of neonatal cardioprotection by rIPC observed in the clinical trial by Jones et al ${ }^{14}$ and, to our knowledge, is the first study to investigate the acute functional and metabolic effects of rIPC on the neonatal heart. Previous studies have shown that immature hearts from various species are neither harmed nor consistently protected by local ischemic
Neonatal

Infarct size of left ventricle

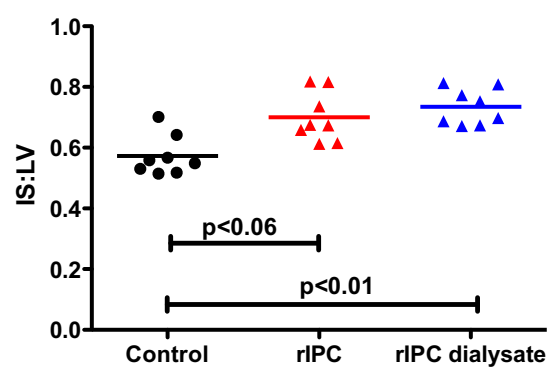

Adult

Infarct size of left ventricle

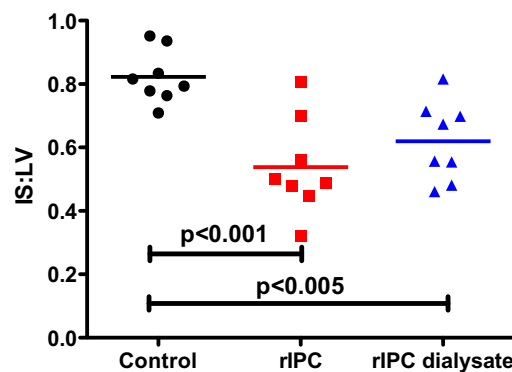

FIGURE 4. Infarct size. $r I P C$, Remote ischemic preconditioning; $L V$, left ventricular. 
preconditioning. However, the present study has shown unexpected deleterious effects of rIPC in the neonatal rabbit heart. Although the results in the adult hearts were consistent with our previous findings, ${ }^{15}$ the infarct size was larger and functional recovery was poorer in the neonatal hearts subjected to either rIPC in vivo or when treated with dialysate from remotely preconditioned adult rabbits. This detrimental effect was associated with increased glycolytic flux during the early reperfusion period in the neonatal myocardium, although the opposite will be seen in association with cardioprotection in the adult heart. However, no certain causality between the changes in glycolytic flux and cardioprotection has been established.

In addition to the divergent responses to rIPC, neonatal and adult hearts displayed important differences in the responses to IR injury alone. Newborn and adult hearts in all groups showed impaired systolic function after prolonged ischemia; however, the neonatal control hearts regained approximately $50 \%$ of baseline LVDP during reperfusion. In contrast, the adult control hearts regained less than $10 \%$ of the baseline LVDP during reperfusion. The negative effect of rIPC and dialysate on neonatal LV function appeared even before the IR injury, indicating that this detrimental effect-although apparently emphasized by IR injury-also affects the unstressed neonatal heart. Furthermore, the infarct size in adult control hearts was significantly larger than that observed in the neonatal control hearts, suggesting that the neonatal heart has an inherent pre-existing level of protection that is clearly adversely modified by additional rIPC.

The present study was not designed to examine the mechanisms of the adverse effects of rIPC. However, some insights into the possible modification of responses can be seen from our data on glycolytic flux. The neonatal hearts, being highly dependent on a carbohydrate metabolism, had an expected higher glycolytic flux throughout the protocol than the adult hearts. However, although adult hearts exposed to rIPC exhibited reduced glycolytic flux in early reperfusion, the neonatal hearts treated with in vivo rIPC had increased glycolytic flux compared with the controls. Although speculative, it appears that rIPC in the neonatal heart increases the need for adenosine triphosphate production through glycolysis during early reperfusion but that a reduction in the glycolytic rate appears to be associated with the protective effect in the adult heart.

Irrespective of the mechanisms, our data are important because they raise questions about the advisability of rIPC as an additional cardioprotective strategy in the early neonatal period for children undergoing cardiac surgery. Because of its simplicity and apparent lack of adverse effects, rIPC has rapidly translated to clinical trials of older patients. However, we would strongly advise that additional mechanistic studies of the detailed metabolism in the neonatal heart be performed before widespread clinical use is initiated. Furthermore, because congestive heart failure and hypertrophic cardiomyopathy seem to induce a reversion of the myocardial metabolism toward a more immature pattern, ${ }^{17,18}$ these finding could have implications regarding the utility of rIPC in other patient groups.

\section{Study Limitations}

Isolated heart preparations provide the possibility of studying the effects of interventions and avoiding extrinsic factors that could influence the outcome. In the present study, the model allowed us to compare the direct effect on neonatal and adult rabbit hearts of dialysate from remotely preconditioned adult rabbits with that of hearts from in vivo preconditioned and control rabbits. However, this ability to study an isolated effect on the heart is gained at the expense of eliminating the physiologic context, and the model might not completely reflect how the bioactive substances from the dialysate would affect the heart in vivo. Furthermore, the dialysate was diluted to $10 \%$ of the in vivo concentration and the dialyzation itself-although preserving the substances protecting the adult hearts-could have eliminated the cardioprotective substances acting on the neonatal heart. Nevertheless, the dual-string design assessing both remote ischemic conditioning dialysate and in vivo rIPC and showing similar effects for the 2 interventions supports the hypothesis that the difficulties in achieving neonatal cardioprotection are related to the heart itself, rather than the signaling mechanisms of rIPC. However, most neonatal IR injury models, including the present one, have been more or less directly copied from adult models, and the window of potential cardioprotection might be different in the neonatal heart (eg, shorter or longer ischemia periods, delayed response).

Finally, the increased infarct size observed in the in vivo remotely preconditioned neonatal hearts was only borderline significant. Although an infarct-increasing effect of rIPC in the neonatal hearts was supported by the significantly larger infarcts in the dialysate group, the increase appeared to be small and most likely of limited clinical significance.

\section{Clinical Perspectives}

Our data have several important implications for future studies of rIPC in neonates and young infants. Our observations of impaired myocardial performance and increased infract size mandate that any clinical study should include a careful evaluation of the effects of the rIPC stimulus itself, before inclusion as a potential therapy in clinical trials.

\section{CONCLUSIONS}

The neonatal heart is inherently more resistant to IR injury than the adult heart. However, this intrinsic protection was reversed when rIPC was performed in vivo, and similar detrimental effects were observed if isolated newborn rabbit 
hearts were perfused with dialysate from remotely preconditioned adult rabbits. More studies are clearly required to better understand the mechanisms of these findings and the ontogeny of early adaptation, before the translation of rIPC to clinical studies.

However, untreated isolated newborn rabbit hearts developed smaller infarcts after IR than did the untreated adult hearts, indicating that the neonatal heart is inherently more resistant to IR injury.

\section{References}

1. Bull C, Cooper J, Stark J. Cardioplegic protection of the child's heart. J Thorac Cardiovasc Surg. 1984;88:287-93.

2. Julia P, Young HH, Buckberg GD, Kofsky ER, Bugyi HI. Studies of myocardial protection in the immature heart. II. Evidence for importance of amino acid metabolism in tolerance to ischemia. J Thorac Cardiovasc Surg. 1990;100: 888-95.

3. Cheung MM, Kharbanda RK, Konstantinov IE, Shimizu M, Frndova H, Li J, et al. Randomized controlled trial of the effects of remote ischemic preconditioning on children undergoing cardiac surgery: first clinical application in humans. J Am Coll Cardiol. 2006; 47:2277-82.

4. Venugopal V, Hausenloy DJ, Ludman A, Di Salvo C, Kolvekar S, Yap J, et al. Remote ischaemic preconditioning reduces myocardial injury in patients undergoing cardiac surgery with cold-blood cardioplegia: a randomised controlled trial. Heart. 2009;95:1567-71.

5. Awad WI, Shattock MJ, Chambers DJ. Ischemic preconditioning in immature myocardium. Circulation. 1998;98(19 suppl):II206-13.

6. Ostadal B, Ostadalova I, Dhalla NS. Development of cardiac sensitivity to oxygen deficiency: comparative and ontogenetic aspects. Physiol Rev. 1999; 79:635-59.
7. Bin Z, Tiehu Y, Su M, Cun L. Absence of apparent cardioprotection following ischemic preconditioning in immature rabbit hearts. Heart Lung Circ. 2003 12:39-43.

8. Zhu B, Min S, Long C, Ye T. Ischemic preconditioning in immature hearts: mechanism and compatibility with cardioplegia. Chin Med J. 2003;116:253-7.

9. Ostadalova I, Ostadal B, Kolar F, Parratt JR, Wilson S. Tolerance to ischaemia and ischaemic preconditioning in neonatal rat heart. J Mol Cell Cardiol. 1998;30:857-65.

10. Webster KA, Discher DJ, Bishopric NH. Cardioprotection in an in vitro model of hypoxic preconditioning. J Mol Cell Cardiol. 1995;27:453-8.

11. Ostadalova I, Ostadal B, Kolar F. Effect of prenatal hypoxia on contractile performance and responsiveness to $\mathrm{Ca} 2+$ in the isolated perinatal rat heart Physiol Res Acad Sci Bohem. 1995;44:135-7.

12. Ascuitto RJ, Ross-Ascuitto NT. Substrate metabolism in the developing heart Semin Perinatol. 1996;20:542-63.

13. Vogt AM, Poolman M, Ackermann C, Yildiz M, Schoels W, Fell DA, et al Regulation of glycolytic flux in ischemic preconditioning: a study employing metabolic control analysis. J Biol Chem. 2002;277:24411-9.

14. Jones BO, Pepe S, Sheeran FL, Donath S, Hardy P, Shekerdemian L, et al Remote ischemic preconditioning in cyanosed neonates undergoing cardiopulmonary bypass: a randomized controlled trial. J Thorac Cardiovasc Surg. February 13, 2013 [Epub ahead of print].

15. Shimizu M, Tropak M, Diaz RJ, Suto F, Surendra H, Kuzmin E, et al Transient limb ischaemia remotely preconditions through a humoral mechanism acting directly on the myocardium: evidence suggesting cross-species protection. Clin Sci (Lond). 2009;117:191-200.

16. Rovetto MJ, Whitmer JT, Neely JR. Comparison of the effects of anoxia and whole heart ischemia on carbohydrate utilization in isolated working rat hearts Circulation Res. 1973;32:699-711.

17. Depre C, Shipley GL, Chen W, Han Q, Doenst T, Moore ML, et al Unloaded heart in vivo replicates fetal gene expression of cardiac hypertrophy. Nat Med. 1998;4:1269-75.

18. Razeghi P, Young ME, Alcorn JL, Moravec CS, Frazier OH, Taegtmeyer H Metabolic gene expression in fetal and failing human heart. Circulation. 2001; 104:2923-31. 